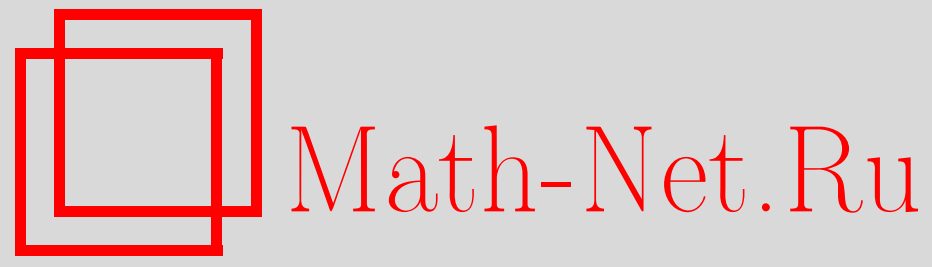

В. А. Родин, Смещение пространств с помощью преобразований Харди и Беллмана, Функи. анализ и его прил., 2000, том 34, выпуск 2, 89-91

DOI: https://doi.org/10.4213/faa305

Использование Общероссийского математического портала MathNet.Ru подразумевает, что вы прочитали и согласны с пользовательским соглашением

http://www . mathnet.ru/rus/agreement

Параметры загрузки:

IP : 54.224 .135 .184

26 апреля 2023 г., 11:54:01

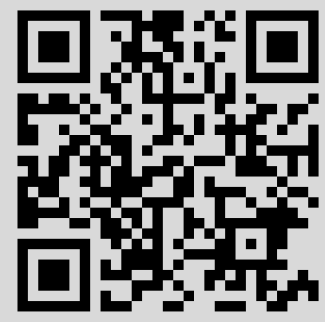


УДК 517.5

\title{
Смещение пространств с помощью преобразований Харди и Беллмана
}

\author{
(c) 2000. В. А. Родин
}

Преобразование тригонометрического ряда с помощью замены его коэффициентов на их арифметические средние порождает оператор Харди [1]: для функции $f \in L_{1}$, имеющей косинус-ряд Фурье

положим

$$
\sum_{k=1}^{\infty} a_{k} \cos k x \quad\left(\bar{a}=\left\{a_{1}, a_{2}, \ldots\right\}\right),
$$

$$
H(f)(x) \sim \sum_{k=1}^{\infty} H_{k}(\bar{a}) \cos k x, \quad \text { где } H_{k}(\bar{a})=\frac{1}{k} \sum_{i=1}^{k} a_{i} .
$$

Для функции $g \in L_{p}, p>1$, имеющей ряд Фурье вида $(1)$, определим оператор Беллмана $B$ следующим образом [2]:

$$
B(g)(x) \sim \sum_{k=1}^{\infty} B_{k}(\bar{a}) \cos k x, \quad \text { где } B_{k}(\bar{a})=\sum_{i=k}^{\infty} \frac{a_{i}}{i} .
$$

Известно, что для $1 \leqslant p<\infty$ пространства $L_{p}$ инвариантны относительно $H$, а $L_{\infty}$ - нет [1]. Для $1<p \leqslant \infty$ эти пространства инвариантны относительно $B$, а пространство $L_{1}$ не инвариантно. Расширяя рамки рассматриваемых пространств, П. Л. Ульянов (см. [3]) поставил задачу о нахождении необходимых и достаточных условий на $N$-функцию в шкале пространств Орлича $L_{N}$, гарантирующих инвариантность данного пространства относительно $H$ и $B$. Эта задача была решена Е. Алшынбаевой [3]. Затем в работах К. Андерсена [4] и О. Я. Берчияна [5] были получены аналогичные результаты для широкого класса симметричных пространств.

Изучение поведения операторов $H$ и $B$ в других пространствах, «близких» к краям шкалы $L_{p}$, а именно в $\operatorname{Re}^{+} H$ и $B M O$, описано в работах Б. И. Голубова $[6,8]$. Подводя итоги изложенному выше, можно заметить, что для различных пространств выяснялись условия их инвариантности относительно преобразований Харди и Беллмана или доказывалась их неинвариантность. Не инвариантные относительно оператора Харди пространства «находились около» $L_{\infty}, \mathrm{a}$ не инвариантные относительно оператора Беллмана - около пространства $L_{1}$. При этом закон «искажения» пространств (например, ВMO) не исследовался. В настоящей работе для некоторых классов симметричных пространств, в частности для пространств Орлича, а также для пространств $B M O$ и $\operatorname{Re}^{+} H$ установлена точная граница смещения пространств — образов операторов $H$ и $B$ относительно пространств их определения.

Далее все функции из области определения операторов будем считать суммируемыми на $[0, \pi]$, продолженными четным образом на $[-\pi, \pi]$ и $2 \pi$-периодическими. Пусть $\varphi$ и $\psi$ - вогнутые возрастающие на $[0, \pi]$ функции. Пространства Лоренца $\Lambda(\varphi)$ и Марцинкевича $\mathrm{M}(\psi)$, так же, как пространство Орлича $L_{N}$, 
являются важнейшими представителями класса симметричных пространств. Определение симметричных пространств см. в [7].

Теорема 1. (1) Для $0<\beta<\infty$ оператор $H$ ограниченно действует из пространства Мариинкевича $\mathrm{M}\left(\tau \ln ^{\beta}(\right.$ ел/ $\left.\tau)\right)$ в пространство Мариинкевича $\mathrm{M}\left(\tau \ln ^{1+\beta}(e \pi / \tau)\right)$.

(2) Oператор $Н$ является неограниченньм как оператор из $\mathrm{M}\left(\tau \ln ^{\beta}(\right.$ ел $\left./ \tau)\right)$ в любое симметричное пространство, строго вложенное в $\mathrm{M}\left(\tau \ln ^{1+\beta}(\right.$ ел $\left./ \tau)\right)$, m. е. такое, ито $E \varsubsetneqq \mathrm{M}\left(\tau \ln ^{1+\beta}(e \pi / \tau)\right)$.

Известно (см. [10, с. 248]), что пространство Орлича с быстро растущей $N$-функцией совпадает с пространством Марцинкевича. Поэтому из теоремы 1 в качестве следствия получаем такое утверждение:

Теорема 2. (1) Для $0<\alpha<\infty$ оператор $H$ ограниченно действует из пространства Орлича $L_{N(\alpha)}$, где $(N(\alpha))(u)=\exp \left(|u|^{\alpha}\right)-1$, в пространство Орлича $L_{\bar{N}(\alpha)}$, где $(\bar{N}(\alpha))(u)=\exp \left(|u|^{\alpha /(\alpha+1)}\right)-1$.

(2) Oператор $H$ является неограниченньл как оператор из $L_{N(\alpha)}$ в любое симметричное пространство $E$, если $E \varsubsetneqq L_{\bar{N}}(\alpha)$.

Для случая $\alpha=1$ данное утверждение доказано в работе [10]. Утверждения теорем 1 и 2 справедливы и в предельном случае $\beta=0$ и $\alpha=\infty$.

Tеорема 3. (1) Onератор $H$ ограниченно действует из $L_{\infty}$ в $\mathrm{M}(\tau \ln (e \pi / \tau))$ ( или $\left.в \quad L_{N} \quad c N(u)=\exp |u|-1\right)$.

(2) Oператор $H$ является неограниченньл как оператор из $L_{\infty}$ в любое симметричное пространство , если $E \varsubsetneqq \mathrm{M}(\tau \ln (e \pi / \tau))$.

Из теоремы 2 можно получить утверждения о поведении оператора $H$ в пространстве $B M O$.

Tеорема 4. Onератор $H$ ограниченно действует из ВМО в пространство Орлича $L_{\Phi}$ с $\Phi(u)=\exp |u|^{1 / 2}-1$ и является неограниченныл как оператор из ВМО в любое симметричное пространство , если $E \varsubsetneqq L_{\Phi}$.

ЗАмЕчАниЕ. Из теоремы 4 следует неинвариантность пространства $B M O$ относительно оператора $H$, ранее доказанная Голубовым [6]. Действительно, если $E=L_{N}$, где $N(u)=\exp |u|-1$, то, поскольку $L_{N} \subset L_{\Phi}$, в силу теоремы 4 оператор $H$ является неограниченным оператором из $B M O$ в $L_{N}$. Неравенство Джона-Ниренберга (см. [11, с. 231]) можно интерпретировать как строгое вложение $B M O \varsubsetneqq L_{N}$; следовательно, $H$ не является ограниченным оператором из $B M O$ в $B M O$. Операторы $H$ и $B$ в определенном смысле (см. [6]) двойственны, а пространство Марцинкевича является сопряженным к пространству Лоренца (см. [8, с. 54]). В связи с этим для оператора Беллмана справедливы результаты, двойственные к теоремам 1-4.

Сформулируем один из них.

Теорема 5. (1) Для $0 \leqslant \beta<\infty$ оператор $B$ ограниченно действует из пространства Орлича $L\left(\ln ^{+} L\right)^{1+\beta}$ в пространство Орлича $L\left(\ln ^{+} L\right)^{\beta}$.

(2) Oператор $B$ является неограниченным как оператор из симметричного пространства $F$ в $L\left(\ln ^{+} L\right)^{\beta}$, если $F \supseteqq L\left(\ln ^{+} L\right)^{1+\beta}$.

Автор выражает благодарность Б. И. Голубову за предоставленную им информацию и внимание к работе. 


\title{
ЛИТЕРАТУРА
}

1. Hardy G. H. Messenger of Math., 58, 50-52 (1928). 2. Bellman R. Bull. Amer. Math. Soc., 50, 741-744 (1994). 3. Алшынбаева E. Матем. заметки, 25, № 5, 645-651 (1979). 4. Andersen K. C. Pacif. J. Math., 100, No. 2, 243-248 (1982). 5. Берчиян О. Я. Матем. заметки, 53, № 4, 3-12 (1993). 6. Голубов Б. И. Некоторые проблемы современной математики и их приложения к задачам физики и механики. МФТИ, М., 1995, с. 56-64. 7. Крейн С. Г., Петунин Ю. И., Семенов Е. М. Интерполяция линейных операторов. Наука, М, 1978. 8. Голубов В. И. Матем. сборник, 185, № 11, 31-40 (1994). 9. Родин B. A. Phystech journal, 3, No. 4, 79-83 (1997). 10. Андриенко В. А. В кн.: Математический анализ. 1970, Итоги науки, ВИНИТИ, М., 1971, с. 203-262. 11. Гарнетт Дж. Ограниченные аналитические функции. Мир, М., 1984.

Воронежский госуниверситет,

Поступило в редакцию математический факультет

18 мая 1998 г.

\section{УДК 517.5}

\section{Совместный спектральный радиус и инвариантные подпространства}

\author{
(c) 2000. Ю. В. ТуровСКий, В. С. Шульман
}

Пусть $M$ - ограниченное множество элементов (комплексной) нормированной алгебры $A,\|M\|=\sup \{\|a\|: a \in M\}$ и $M^{n}-$ множество всех произведений по $n$ элементов из $M$. Число $\rho(M)=\inf \left\|M^{n}\right\|^{1 / n}$ называется [1] спектральньлм радиусом множества $M$. Если $\rho(M)=0$, то $M$ называется квазинильпотентнылм. Множество называется конечно (компактно) квазинильпотентнылм, если все его конечные (соответственно предкомпактные) подмножества квазинильпотентны. Для множества операторов $M \subset B(\mathfrak{X})$ мы также рассматриваем существенный спектральный радиус $\rho_{e}(M)$, равный спектральному радиусу образа множества $M$ в алгебре Калкина $B(\mathfrak{X}) / K(\mathfrak{X})$, где $B(\mathfrak{X})$ - алгебра всех ограниченных линейных операторов в банаховом пространстве $\mathfrak{X}$ и $K(\mathfrak{X})$ - ее идеал, состоящий из всех компактных операторов. Первые применения этих понятий в теории инвариантных подпространств были найдены в [2], где установлено, что алгебра вольтерровых (т. е. компактных квазинильпотентных) операторов имеет гиперинвариантное (инвариантное относительно всех операторов из алгебры и ее коммутанта) подпространство (ГИП), и в [3] (подробнее см. в [4]), где, в частности, доказано, что два коммутирующих триангулируемых семейства компактных операторов триангулируемы одновременно. Развитие этой техники в [5] позволило первому автору решить проблему вольтерровой полугруппы, т.е. доказать, что любая (мультипликативная) полугруппа вольтерровых операторов имеет (здесь и далее подразумевается нетривиальное) ГИП. Мы приводим другие приложения в теории инвариантных подпространств.

Теорема 1. Пусть $M \subset B(\mathfrak{X})$ - предкомпактное множество операторов, такое, что $\rho_{e}(M)<\rho(M)=1$. Если порожденная им полугруппа неограничена, то она имеет ГИП. 